2007s-15

\title{
Approche expérimentale de l'incidence de la fiscalité sur l'offre de travail : \\ Une étude comparative des systèmes d'imposition
}

David Masclet. Claude Montmarauette

\begin{tabular}{c}
\hline Série Scientifique \\
Scientific Series
\end{tabular}

\section{Montréal}

Juillet 2007

(C) 2007 David Masclet, Claude Montmarquette. Tous droits réservés. All rights reserved. Reproduction partielle permise avec citation du document source, incluant la notice (C).

Short sections may be quoted without explicit permission, if full credit, including (c) notice, is given to the source.
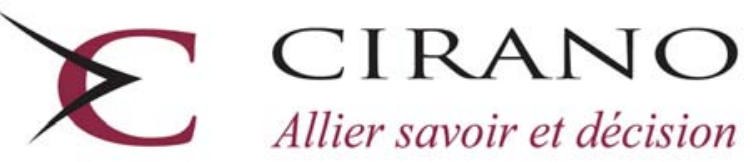

Allier savoir et décision

Centre interuniversitaire de recherche en analyse des organisations 


\section{CIRANO}

Le CIRANO est un organisme sans but lucratif constitué en vertu de la Loi des compagnies du Québec. Le financement de son infrastructure et de ses activités de recherche provient des cotisations de ses organisations-membres, d'une subvention d'infrastructure du Ministère du Développement économique et régional et de la Recherche, de même que des subventions et mandats obtenus par ses équipes de recherche.

CIRANO is a private non-profit organization incorporated under the Québec Companies Act. Its infrastructure and research activities are funded through fees paid by member organizations, an infrastructure grant from the Ministère du Développement économique et régional et de la Recherche, and grants and research mandates obtained by its research teams.

\section{Les partenaires du CIRANO}

Partenaire majeur

Ministère du Développement économique, de l'Innovation et de l'Exportation

\section{Partenaires corporatifs}

\section{Alcan inc.}

Banque de développement du Canada

Banque du Canada

Banque Laurentienne du Canada

Banque Nationale du Canada

Banque Royale du Canada

Banque Scotia

Bell Canada

BMO Groupe financier

Bourse de Montréal

Caisse de dépôt et placement du Québec

DMR Conseil

Fédération des caisses Desjardins du Québec

Gaz de France

Gaz Métro

Hydro-Québec

Industrie Canada

Investissements PSP

Ministère des Finances du Québec

Raymond Chabot Grant Thornton

State Street Global Advisors

Transat A.T.

Ville de Montréal

\section{Partenaires universitaires}

École Polytechnique de Montréal

HEC Montréal

McGill University

Université Concordia

Université de Montréal

Université de Sherbrooke

Université du Québec

Université du Québec à Montréal

Université Laval

Le CIRANO collabore avec de nombreux centres et chaires de recherche universitaires dont on peut consulter la liste sur son site web.

Les cahiers de la série scientifique (CS) visent à rendre accessibles des résultats de recherche effectuée au CIRANO afin de susciter échanges et commentaires. Ces cahiers sont écrits dans le style des publications scientifiques. Les idées et les opinions émises sont sous l'unique responsabilité des auteurs et ne représentent pas nécessairement les positions du CIRANO ou de ses partenaires.

This paper presents research carried out at CIRANO and aims at encouraging discussion and comment. The observations and viewpoints expressed are the sole responsibility of the authors. They do not necessarily represent positions of CIRANO or its partners. 


\title{
Approche expérimentale de l'incidence de la fiscalité sur l'offre de travail :
}

\section{Une étude comparative des systèmes d'imposition ${ }^{*}$}

\author{
David Masclet ${ }^{\dagger}$, Claude Montmarquette ${ }^{\ddagger}$
}

\begin{abstract}
Résumé/ Abstract
L'enjeu de cet article est de savoir si l'incidence fiscale sur l'offre de travail et sur les rentrées fiscales est identique selon le système d'imposition en vigueur. Ainsi, un système progressif décourage-t-il davantage l'offre de travail qu'un système proportionnel ? Quel système de taxation permet à l'état de remplir au mieux son rôle redistributif ? Afin de répondre à ces questions, l'approche mobilisée est l'économie expérimentale. Trois traitements ont été réalisés. Dans un premier traitement, les participants doivent réaliser un effort réel sous un système d'imposition proportionnel. Dans un second traitement, le système d'imposition en vigueur est un système progressif équivalent. Enfin dans un troisième traitement, les participants sont confrontés successivement à plusieurs systèmes d'imposition. Ce dernier traitement permet d'étudier l'incidence de changement de système sur les décisions individuelles. Nos résultats expérimentaux montrent que les effets désincitatifs sur l'offre de travail sont plus importants sous un système progressif que sous un système proportionnel sans contexte. De plus le système proportionnel sans contexte permet des rentrées fiscales plus importantes. Enfin, un autre résultat intéressant de notre étude est que les individus ne sont pas uniquement influencés par leur propre système fiscal mais également par leurs expériences fiscales précédentes.
\end{abstract}

Mots clés : systèmes fiscaux, offre de travail et recettes fiscales, étude expérimentale

This experiment evaluates various systems of taxation on the number of real tasks performed by participants and in terms of fiscal income generated to help government in its redistributive goal. The first treatment concerns a proportional system of taxation. The second treatment considers an equivalent progressive income tax system. Finally, participants are submitted sequentially to different flat tax rates. This last treatment evaluates the reaction of participants to fiscal changes. Our results support clearly that a progressive taxation system reduces work effort relative to a single flat tax. The single flat tax system yields more fiscal income. The results also show that individuals are affected by their own tax system and by the other forms of taxation they experienced before.

Keywords: fiscal systems, work effort, fiscal income, experimental study

Codes JEL : C91 H30 J22

\footnotetext{
* Nous remercions Bruno Girard, Nathalie Viennot-Briot et Romain Zeillinger pour la programmation et la réalisation des expériences, ainsi que le personnel du CIRANO et du LUBC3E. Les commentaires, remarques et suggestions d'un arbitre anonyme et des participants aux $7^{\text {emes }}$ Journées d'Economie Expérimentale, Rennes 2005 ont été appréciés pour rédiger la version finale de ce texte. Nous assumons seuls la responsabilité du contenu de cette recherche.

${ }^{\dagger}$ Auteur pour la correspondance. CREM, CIRANO et Université Rennes 1, David.Masclet@univ-rennes1.fr.

† CIRANO et Université de Montréal, Claude.Montmarquette@cirano.qc.ca.
} 


\section{Introduction}

Les taux de prélèvement dans les pays développés représentent en moyenne $40 \%$ du PIB. ${ }^{1}$ Or si la fiscalité est indispensable pour assurer le fonctionnement de l'Etat, elle peut faire peser un certain nombre de menaces sur les performances de l'économie. En effet, des niveaux élevés d'imposition peuvent conduire les contribuables à modifier leur comportement économique de plusieurs façons. Ainsi, les charges fiscales peuvent inciter certains individus à frauder (Cowel, 1985; Henry, 1983) ou à rechercher à l'étranger des conditions fiscales plus favorables (Cowel, 1985). Les individus peuvent également être tentés par l'économie souterraine de façon à réduire leur charge fiscale ou à préserver leurs prestations sociales (Henry, 1983; Cowel, 1985; Fortin, Garneau, Lacroix, Lemieux et Montmarquette, 1996; Fortin, Joubert et Lacroix, 2005). ${ }^{2}$ Enfin, comme l'ont montré plusieurs travaux empiriques dont certaines études expérimentales, la fiscalité peut également avoir des effets désincitatifs et décourager l'offre de travail des individus. ${ }^{3}$ Hausman (1981) et Ashworth et Ulph (1981) observent qu'une réduction des taxes conduit à un accroissement de l'offre de travail. D’autres études mettent en exergue le fait que les taxes n'affectent pas de la même façon tous les individus. Ainsi Sillamaa et Veal (2000) observent, à partir d'une enquête sur les effets de la réforme sur les taxes fédérales en 1988 au Canada, que la sensibilité à un changement de taux marginal de taxation dépend de facteurs tels que le type d'emploi, l’âge ou l'importance des revenus perçus. Les catégories de population les plus âgées, les travailleurs indépendants ou les catégories avec de hauts revenus seraient des catégories très sensibles aux changements de taxes. ${ }^{4}$ Slemrod $(1996,1998)$ obtient des résultats similaires sur données américaines. Des études expérimentales ont également étudié les effets de la fiscalité sur l'offre de travail. Swenson (1988) a réalisé une expérience en effort réel où les sujets de l'expérience sont confrontés à 5 taux de taxe différents: 12\%, 28\%, 50\%, 73\% ou 87\% ${ }^{5}$. L'auteur observe que l'offre de travail diminue à partir du deuxième taux le plus bas (28\%). Sillamaa (1999a) a répliqué l'étude de Swenson et obtient des résultats similaires. Sutter et Weck Hannemann (2002) ont étudié, quant à eux, l'effet d'une variation du taux de taxation sur l'offre de travail en endogénéisant le choix du taux de taxation. ${ }^{6}$ Les auteurs observent également que l'effort diminue lorsque le taux de taxe augmente. Dans deux études récentes, Lévy-Garboua, Masclet et Montmarquette (2005a, 2005b) ont confirmé l'ensemble de ces résultats et mettent en 
évidence l'existence d'une courbe de Laffer avec un point de retournement situé entre 50 et $65 \%{ }^{7}$

L'enjeu principal de cet article est d'étudier si l'incidence fiscale sur l'offre de travail et sur les rentrées fiscales est identique selon le système d'imposition. En effet, il existe une grande variété de systèmes fiscaux. Si le système d'impôt progressif sur le revenu est actuellement le plus répandu, plusieurs pays ont récemment opté pour une taxe unique proportionnelle au revenu. Ainsi on peut citer notamment certains pays d'Europe de l'est comme la Slovaquie avec un taux de 19\%, la Roumanie avec un taux de 16\%, la Russie et l'Ukraine avec un taux de $13 \%$, les pays baltes Estonie, Lettonie et Lituanie avec des taux respectifs de 24\%, 25\% et 33\%, la Serbie avec un taux de 14\%. Aux États-Unis, le système national d'imposition sur le revenu est progressif toutefois cinq États (l'Illinois, le Massachusetts, le Michigan et la Pennsylvanie) ont instauré un impôt proportionnel d'État sur le revenu. ${ }^{8}$ Enfin au Canada, l'Alberta a opté pour un taux unique. En France, d'une certaine manière, la fiscalité directe est à la fois progressive avec l'impôt sur le revenu et à taux unique avec l'Impôt sur les Sociétés et la CSG. Au niveau de la fiscalité directe, le système français a donc une composante "taux unique" relativement importante par rapport à la composante à "taux progressif". Actuellement, la Grèce, la République Tchèque et la Croatie ont prévu d'instaurer ce type d'imposition.

Historiquement, le système d'imposition le plus ancien est le système proportionnel à taux unique qui est apparu comme une amélioration fiscale, par rapport à une situation héritée de l'ancien régime français où seule une partie de la population payait l'impôt (le tiers état tandis que la population composée du clergé et de la noblesse en était exemptée). ${ }^{9}$ A partir du XIXe siècle, des systèmes d'imposition à taux unique ont ainsi été progressivement mis en place dans la plupart des pays européens. Ce n'est qu'au début du XXe siècle, après la Première Guerre mondiale, que de nombreux pays remplacèrent ce système par un système d'imposition progressif visant à fiancer les systèmes sociaux créés à l'époque. Depuis, un certain nombre d'études économiques ont montré les limites de ce système progressif. La raison principalement évoquée est qu'un système progressif comporte des effets désincitatifs, notamment pour les tranches d'impositions les plus élevées où le taux marginal d'imposition peut être très important, ce qui, en décourageant l'offre de travail, conduirait à réduire les recettes fiscales. Ainsi, la Russie a vu les recettes de son impôt sur le revenu augmenter successivement de 25,2\%, 24,6\% et 15,2\% les 3 années qui ont suivi l'instauration de l'impôt à taux unique, soit une hausse totale 
de près de 80 \%. La deuxième raison souvent avancée est qu'un système progressif conduirait à la fois à la création de niches fiscales où se réfugient les acteurs économiques qui se trouvent sur la tranche d'imposition la plus haute et également inciterait à l'évasion fiscale.

Nous comparons dans cette étude les effets de deux systèmes d'imposition: un système de taxation proportionnel et un système d'imposition non proportionnel à taux progressif. ${ }^{10}$ Un système progressif décourage-t-il davantage l'offre de travail qu'un système proportionnel? Par ailleurs, les rentrées fiscales sont-elles plus importantes dans un système proportionnel que dans un système progressif ? Un deuxième enjeu de cet article est d'étudier l'incidence sur l'offre de travail et par voie de conséquence sur les rentrées fiscales d'un changement de système d'imposition. Ainsi les individus réagissent-ils de la même façon selon qu'ils ont toujours été confrontés à un système donné ou qu'ils ont été amenés à changer de système et, ont donc expérimenté d'autres systèmes d'imposition par le passé ? Le contexte fiscal constitué des expériences fiscales passées est-il susceptible d'influencer les décisions de travail des individus ? ?1 $^{11}$

Afin de répondre à ces questions, l'approche mobilisée est l'économie expérimentale. Dans notre expérience, les participants sont regroupés par paires. Chaque paire est constituée de deux participants : un participant A et un participant B. L'expérience est composée de 18 périodes. Chaque période se déroule de la façon suivante: les participants B doivent décider combien de tâches productives ils désirent exercer entre 0 et 52 tâches. Après avoir effectué les tâches, les participants reçoivent leur gain. Le gain du participant B correspond au revenu du travail diminué du taux de prélèvement. Le gain du participant $\mathrm{A}$ est constitué de la partie du revenu prélevée sur le gain du participant B. L'expérience est composée de trois traitements. Dans un premier traitement, le revenu du participant B est soumis à un impôt proportionnel. Dans un second traitement, le système d'imposition est progressif. Dans ces deux traitements, les participants sont confrontés à un seul système d'imposition pendant toute la durée de l'expérience. Cela offre l'avantage de mesurer les incidences du système fiscal indépendamment du contexte fiscal dans la mesure où les participants ne peuvent pas comparer leur situation fiscale avec des situations qu'ils auraient pu expérimenter dans le passé. Dans un troisième traitement, nous relâchons cette contrainte afin d'étudier si les individus peuvent également être influencés par leurs expériences fiscales précédentes. Dans ce traitement, les participants sont 
donc soumis à différents systèmes d'imposition durant l'expérience dont le système proportionnel du premier traitement. La comparaison du traitement 1 et du traitement 3 pour ce même système nous permet donc d'isoler l'incidence du contexte fiscal sur les comportements économiques et les rentrées fiscales.

Notre étude expérimentale s'inspire des travaux expérimentaux de Sillamaa (1999b; Sillamaa (1999c). Sillamaa (1999b) a comparé les effets sur l’offre de travail d'une variation du taux de salaire sous un système proportionnel et sous un système progressif. L'auteur observe que l'effort est plus élevé dans un système proportionnel que dans le système progressif après une augmentation de salaire. Dans une seconde étude, Sillamaa (1999c) compare les effets sur l’offre de travail d'un système non proportionnel selon que le taux marginal de taxation supérieur est nul ou positif. L'auteur conclut que, conformément à la prédiction théorique, le passage au taux marginal de taxation nul incite les agents à accroître leur effort de production. ${ }^{12}$ Notre étude se distingue des travaux de Sillamaa sur plusieurs points. Contrairement à Sillamaa, nous distinguons deux types de participants (participant $\mathrm{A}$ et participant B) et considérons que la part de revenu prélevée sur le gain de B est transférée au participant A qui est le bénéficiaire de ces transferts. Dans cette perspective, nos travaux se rapprochent davantage de ceux de Sutter et Weck-Hannemann (2003) et offrent davantage de réalisme.

Nos résultats expérimentaux montrent que les effets désincitatifs sur l'offre de travail sont plus importants sous un système progressif que sous un système proportionnel. Un autre résultat intéressant de notre étude est que le contexte fiscal affecte également les comportements individuels. En effet, pour un taux donné, les participants choisissent leur offre de travail en tenant compte de leurs expériences fiscales précédentes.

La section 2 fait l'objet de la présentation du protocole expérimental. La section 3 donne les principaux résultats de cette étude tandis que la section 4 conclut cet article.

\section{Le protocole expérimental}

Comme dans les travaux déjà cités, le protocole expérimental présenté dans cette section vise à reproduire l’arbitrage travail/loisir. Les participants à notre expérience sont regroupés par paires constituées d'un participant A et d'un participant B. Les participants conservent le même rôle durant toute l'expérience. Ce rôle est assigné au hasard avant le début de l'expérience et après la lecture des instructions. Les participants ont donc le loisir d'évaluer leur stratégie avant 
que ne débute l'expérience. Le participant $\mathrm{B}$ est appelé à exécuter des tâches en effort réel. Le coût à l'effort est double. D'une part, le participant B doit effectuer une tâche en effort réel et d'autre part en effectuant cette tâche, il renonce au loisir. Etant donné que les individus qui se déplacent pour une expérience ont déjà pris la décision de sacrifier, contre une rémunération potentielle, une partie de leur loisir, nous avons évité de préciser dans les instructions le nombre de périodes que les participants seraient appelés à jouer. Les participants sont toutefois conscients que la durée de leur participation est fonction de leurs décisions sur le nombre de tâches à réaliser. Par ailleurs, comme le coût d'opportunité du travail est un élément important dans le choix du nombre de tâches, nous avons retenu un nombre maximum de 52 tâches possibles par jeu. En effet, il est raisonnable de croire que le coût d'opportunité du travail apparaît plus saillant lorsque le volume de travail est grand. Une tâche consiste à décoder un chiffre à partir d'une grille de lettres aléatoires non répétées qui apparaît à l'écran de l'ordinateur. Le participant A joue un rôle passif dans cette expérience. ${ }^{13}$ Il a le loisir de lire diverses revues mises à sa disposition ou de jouer à différents jeux sur l'ordinateur. ${ }^{14}$ Dans tous les traitements, le revenu du participant $B$ est soumis à taxation et la partie prélevée est transférée au participant A.

L'expérience est composée de 18 périodes. Chaque période se déroule de la façon suivante : i) Dans un premier temps, les sujets A et B observent le niveau de taxe choisi par l’ordinateur qui correspond à la part qui sera prélevée sur le gain de B et transférée au sujet A; ii) Les sujets $B$ doivent ensuite décider combien de tâches productives ils désirent exercer sur l'intervalle [0,52] ; iii) Une fois que les sujets ont décidé du nombre de tâches, ils doivent les effectuer. Une tâche erronée n’est pas payée ; iv) Les sujets reçoivent enfin leurs gains nets respectifs. Chaque tâche réalisée sans erreur procure un gain avant imposition de 100 unités de monnaie expérimentale (UME).

Les traitements de l'expérience se distinguent par le système de taxation en vigueur (système progressif ou système proportionnel). Il est à noter que, comme le niveau de taxes est choisi par l'ordinateur, les bénéficiaires des taxes sont clairement distincts des autorités qui déterminent le niveau d'imposition, ce qui permet d'éviter des effets d'interaction entre les deux types de participants. ${ }^{15}$ Les paragraphes suivants présentent en détail les différents traitements. 


\subsection{Les traitements expérimentaux}

Trois traitements ont été réalisés dans cette expérience. Dans un premier traitement, les participants sont soumis à un système de taxation proportionnel avec un taux unique de 50\%. Ce taux demeure inchangé pendant toute la durée de l'expérience. Ainsi la partie transférée au participant A est de 50\%. Le sujet A est informé qu'il va recevoir une part de 50\% qui sera prélevée sur le gain que le sujet B a obtenu en réalisant des tâches sans erreur. Les sujets ne sont pas informés du nombre de périodes (18) qu’ils devront jouer. Ainsi certains participants peuvent terminer plus tôt l'expérience que d'autres en fonction du nombre de tâches choisies et réalisées sans erreurs par le participant $B$ et de leur vitesse respective d'exécution de ces tâches. Le gain net du sujet A correspond au nombre de tâches réalisées sans erreur par son partenaire B multiplié par le taux de taxe de 50\%. Le gain net du sujet B correspond exactement au gain net de A dans ce traitement.

Dans un deuxième traitement, appelé système de taxation progressif, les participants sont confrontés à un système non proportionnel progressif constitué de trois taux: 28\%, 50\% et 79\%. Ainsi les 20 premières tâches réalisées sans erreur (qui correspondent à une tranche de revenu entre 0 et 2000 UME) sont imposées à un taux de 28\%. Le taux intermédiaire d’imposition est de 50\% pour les dix tâches suivantes (correspondant à une tranche de revenu de 2100 à 3000 UME). Enfin le taux marginal supérieur d'imposition est de 79\% et correspond aux tâches au dessus de la 30ième tâche (ce qui correspond à la tranche de revenu située entre 3100 et 5200 $\mathrm{UME}^{16}$. Les paramètres du système progressif ont été définis à partir des observations du système proportionnel. Plus précisément, les taux du système progressif par tranche d'effort ont été déterminés afin d'obtenir ex ante des recettes fiscales identiques à celles obtenues dans le système proportionnel, toute chose étant égale par ailleurs, c'est à dire en supposant inchangée la répartition des revenus entre les deux traitements. Le critère des rentrées fiscales est le critère généralement retenu pour construire un nouveau système fiscal. Ceci suppose évidemment implicitement que le comportement des individus, c'est-à-dire leur décision d'offre de travail, soit identique dans les deux traitements. Par conséquent, si le choix du système n'affecte pas les décisions d'offre de travail des participants, alors on ne devrait observer aucune différence de rentrées fiscales entre les traitements proportionnel et progressif. Au contraire, si le choix du système influence les décisions des individus, alors les rentrées fiscales devraient être différentes entre les deux traitements. 
Le traitement 3 vise à étudier si le niveau d'effort pour un même système avec un taux unique de 50\% est identique selon que les participants ont été confrontés à ce taux durant toute la durée de l'expérience comme c'est le cas dans le traitement 1 ou si, au contraire, ils ont été soumis à d'autres taux au cours du temps. Dans ce traitement, les participants sont confrontés successivement, sans ordre précis, aux taux suivants: 12, 28, 50 et 79\%. ${ }^{17}$ Chaque taux choisi par l'ordinateur est effectif pendant trois périodes successives. ${ }^{18}$ L'expérience se compose donc de six jeux successifs, chaque jeu étant constitué de trois périodes durant lesquelles un même taux est en vigueur. A chaque nouveau jeu, les participants sont soumis à un nouveau taux qui peut être supérieur ou inférieur au taux précédent.

La comparaison du traitement 1 et du traitement 3 pour le taux de 50\% permet d'isoler

l'effet du contexte. ${ }^{19}$ Si les participants ne sont pas influencés par l'existence des autres taux, alors nous ne devrions pas observer de différence significative entre les traitements 1 et 3 pour le même taux de 50\%. Si au contraire, les participants décident de leur niveau d'effort en tenant compte des taux qu'ils ont expérimentés par le passé, alors les participants devraient fournir un effort différent dans les deux traitements. ${ }^{20}$

\subsection{Paramètres de l'expérience et réalisation de l'expérience}

L'expérience a été réalisée au CIRANO (Centre Interuniversitaire de Recherche en Analyse des Organisations) à l'aide du logiciel REGATE développé par Romain Zeilliger du GATE, Université de Lumière, Lyon 2. Treize sessions ont été réalisées. Pour chaque session, les participants ne jouent qu'un seul des trois traitements présentés plus haut. Au total 142 participants ont été recrutés. Les participants ont été recrutés parmi des étudiants de premier cycle dans plusieurs disciplines. Aucun participant n'avait joué à une expérience semblable auparavant. L'expérience a duré en moyenne 2 h30 et la rémunération moyenne est de 17 dollars canadiens de l'heure. Le taux de change est de 900UME =1,00 \$ canadien.

Le tableau 1 résume les différents traitements réalisés et le nombre de participants impliqués par session: 
Tableau 1: Description des sessions expérimentales

\begin{tabular}{ccc}
\hline \hline Session & Système & $\begin{array}{c}\text { Nombre de } \\
\text { participants }\end{array}$ \\
& & 12 \\
1 & Proportionnel & 10 \\
2 & Proportionnel & 12 \\
3 & Proportionnel & 12 \\
4 & Proportionnel & 10 \\
5 & Progressif & 8 \\
6 & Progressif & 10 \\
7 & Progressif & 12 \\
8 & Progressif & 10 \\
9 & Progressif & 12 \\
10 & Proportionnel avec contexte & 10 \\
11 & Proportionnel avec contexte & 12 \\
12 & Proportionnel avec contexte & 12 \\
13 & Proportionnel avec contexte & 142 \\
\hline \hline
\end{tabular}

\section{Les résultats des données expérimentales}

La première sous-section vise à étudier les incidences de la fiscalité sur l'offre de travail selon que le système d'imposition est proportionnel ou progressif. Dans une deuxième section, nous nous intéressons au rôle du contexte fiscal, c'est-à-dire à l'incidence des expériences fiscales précédentes sur les décisions des participants confrontés à un système donné.

\subsection{Système proportionnel d'imposition versus système non proportionnel progressif}

Le graphique 1 montre l'évolution du nombre moyen de tâches réalisées sans erreur dans le cas d'un système proportionnel et progressif. 
Graphique 1. Évolution du nombre moyen de tâches réalisées sans erreur (traitements 1 et 2)

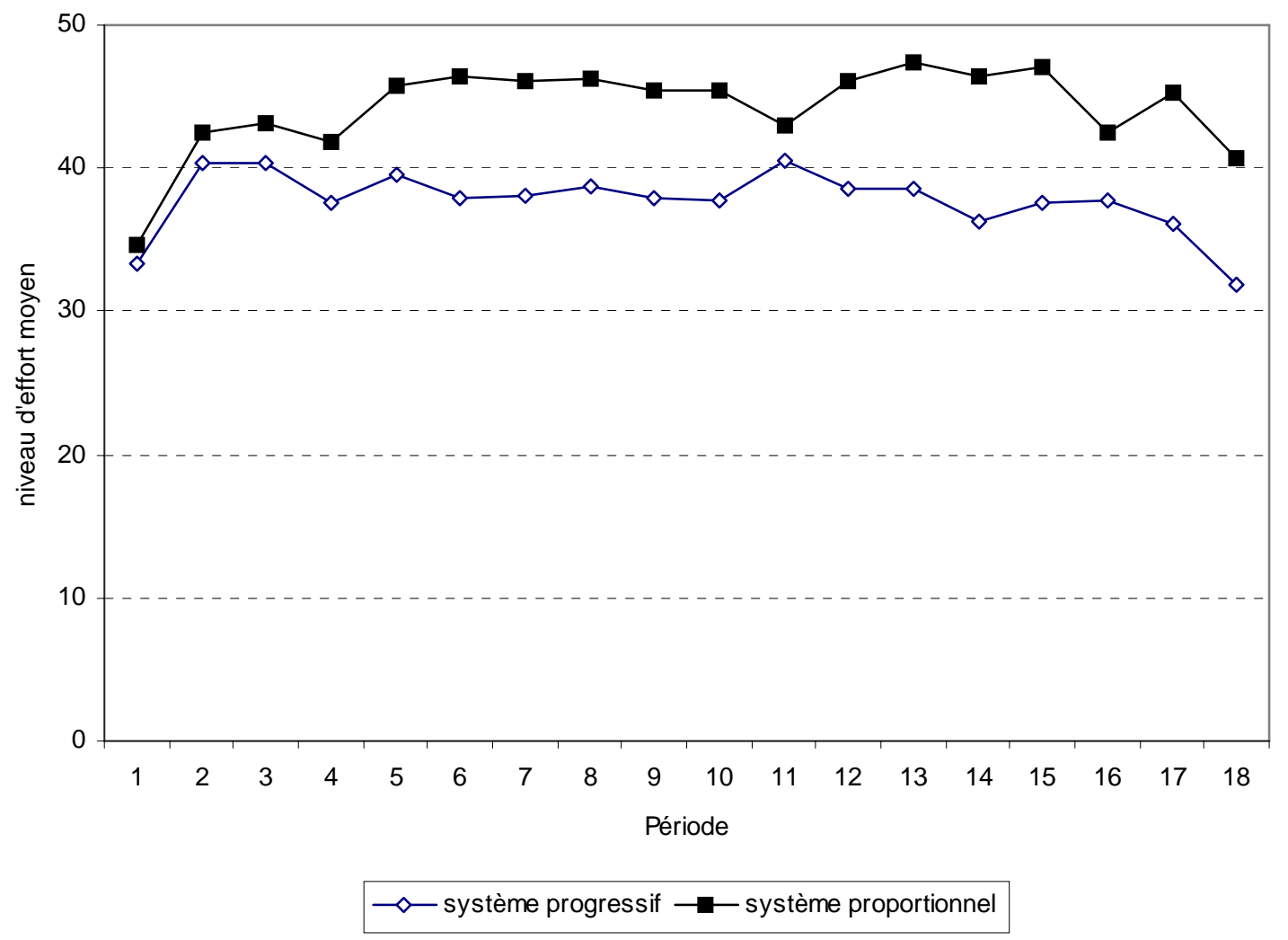

Le graphique 1 indique que les niveaux d'effort réalisés dans les traitements 1 et 2 suivent une évolution similaire. Ainsi, le niveau d'effort augmente au cours des trois premières périodes de l'expérience, puis se stabilise au cours du temps, pour diminuer légèrement lors des dernières périodes. ${ }^{21}$ Si les niveaux d'effort en première période sont quasiment identiques dans les deux traitements (respectivement 34,65 dans le traitement 1 et 33,36 dans le traitement 2), on observe toutefois que le nombre de tâches réalisées sans erreur sur l'ensemble de l'expérience est significativement plus élevé sous un système proportionnel (traitement 1) que sous un système progressif (traitement 2). Ainsi le nombre moyen de tâches réalisées sans erreur sur l'ensemble des périodes du traitement 1 est de 44,21 tâches contre 37,43 dans le traitement 2. Les tests non paramétriques confirment ces observations. Ainsi un test non paramétrique de Mann-Whitney réalisé sur les moyennes individuelles du premier jeu ne montre aucune différence d'effort entre les deux traitements $(\mathrm{z}=-0,250)$. Au contraire, ce même test réalisé sur l'ensemble des périodes 
met en évidence que le niveau d'effort est significativement plus élevé (au niveau de confiance de 1\%) dans le traitement 1 que dans le traitement 2 (test de Mann-Whitney; z = 2,6).

Le graphique 2 donne la répartition par traitement du nombre de tâches réalisées sans erreur sur l'intervalle [0-52]. On observe que la grande majorité des participants choisit le nombre maximal de tâches, c'est à dire 52 tâches, quelque soit le traitement joué. Toutefois la probabilité de choisir 52 tâches est plus élevée dans le traitement 1 que dans le traitement 2. Ainsi la probabilité de choisir le nombre de tâches maximal est de 53\% dans le traitement 1 contre 32\% dans le traitement 2. Si l'on considère maintenant la probabilité de ne fournir aucun effort, cette probabilité est nulle dans le traitement 1 alors qu'elle est d'environ 3\% dans le traitement 2. De même, si l'on considère la proportion de joueurs s'arrêtant avant la 29éme tâche, ce nombre est plus élevé dans le traitement progressif (23,7\%) que dans le traitement proportionnel sans contexte (14\%), ce qui souligne la encore l'effet désincitatif du système progressif. Dans ce cas, il s'agit du passage d'un taux de 28\% au taux de 50\% qui est désincitif sur l'offre de travail.

Enfin, et de façon anecdotique, il semble que les participants aient choisi de se focaliser, sans aucune explication rationnelle, sur les niveaux d'effort correspondant aux dizaines d'unités : 10, 20 30, 40 et 50. Ces résultats peuvent s'interpréter au regard de la définition du point focal de Thomas Schelling 1960, 2007). 
Graphique 2. Distribution des tâches réalisées sans erreur

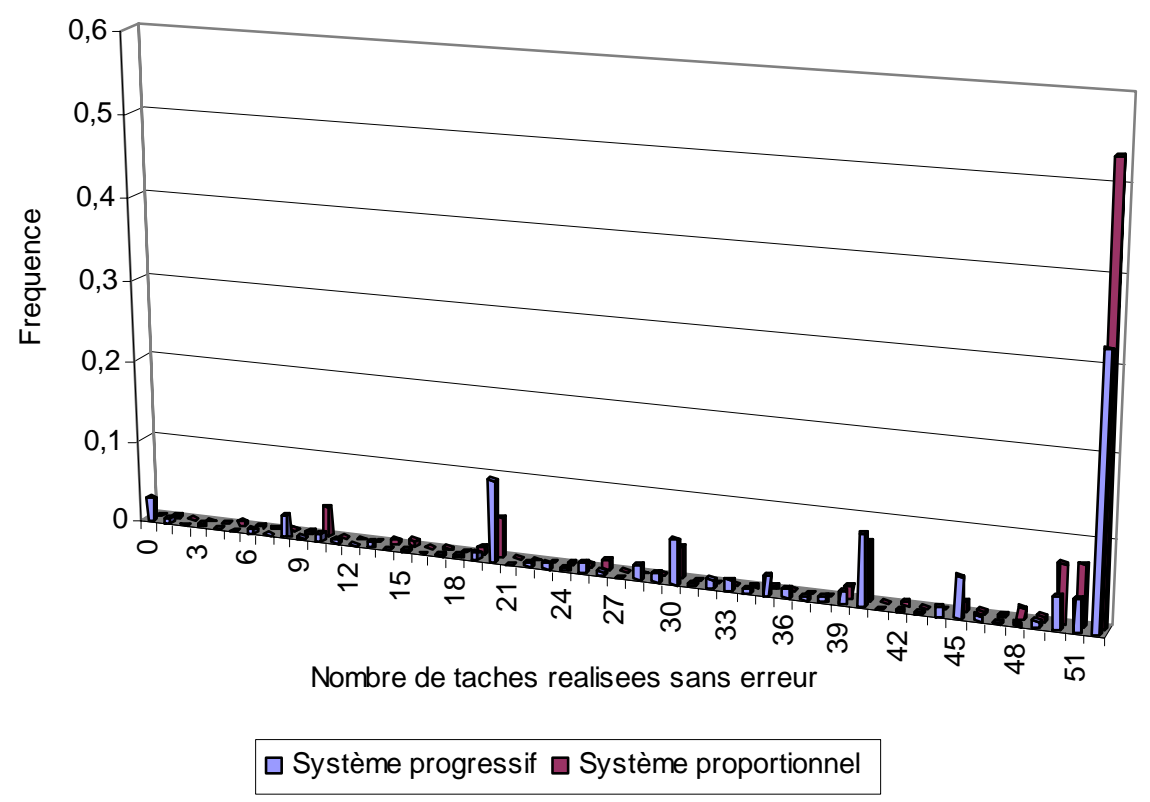

Les résultats précédents montrent que l'incidence fiscale sur l'offre de travail est différente selon le système d'imposition en place. Ainsi le système progressif est davantage désincitatif à l'effort que le système proportionnel. On observe par ailleurs que la répartition des tâches réalisées est différente dans les deux traitements. On peut dès lors s'interroger sur l'impact respectif de chacun de ces systèmes sur les recettes fiscales. En effet l'ajustement dans l'offre de travail selon le système fiscal retenu peut entraîner des rentrées fiscales différentes. Les rentrées fiscales correspondent dans cette expérience au gain du participant A. Nos résultats indiquent que les rentrées fiscales sont significativement plus élevées dans le système proportionnel que dans le système progressif. Alors que le gain du participant A est de 2210 UME dans le traitement 1, il n'est que de 1962 UME dans le traitement 2. Un test de Mann-Whytney réalisé sur les gains individuels moyens indiquent que cette différence est significative $(\mathrm{z}=-2,384)$. En pourcentage, les rentrées fiscales sous le système progressif sont inférieures de $11,2 \%$ à celles obtenues avec le système proportionnel. 


\subsection{Existe-t-il un effet "contexte"?}

Le graphique 3 compare l'évolution du nombre moyen de tâches réalisées sans erreur pour un système proportionnel avec un taux unique de 50\% selon qu'il existe ou pas un contexte, c'est-à-dire selon que les participants sont confrontés à 50\% durant toute l'expérience (traitement 1) ou qu'ils ont pu expérimenter d'autres taux au cours du jeu (traitement 3).

Graphique 3. Évolution du nombre moyen de tâches réalisées sans erreur pour un taux de 50\% (traitements 1 et 3)

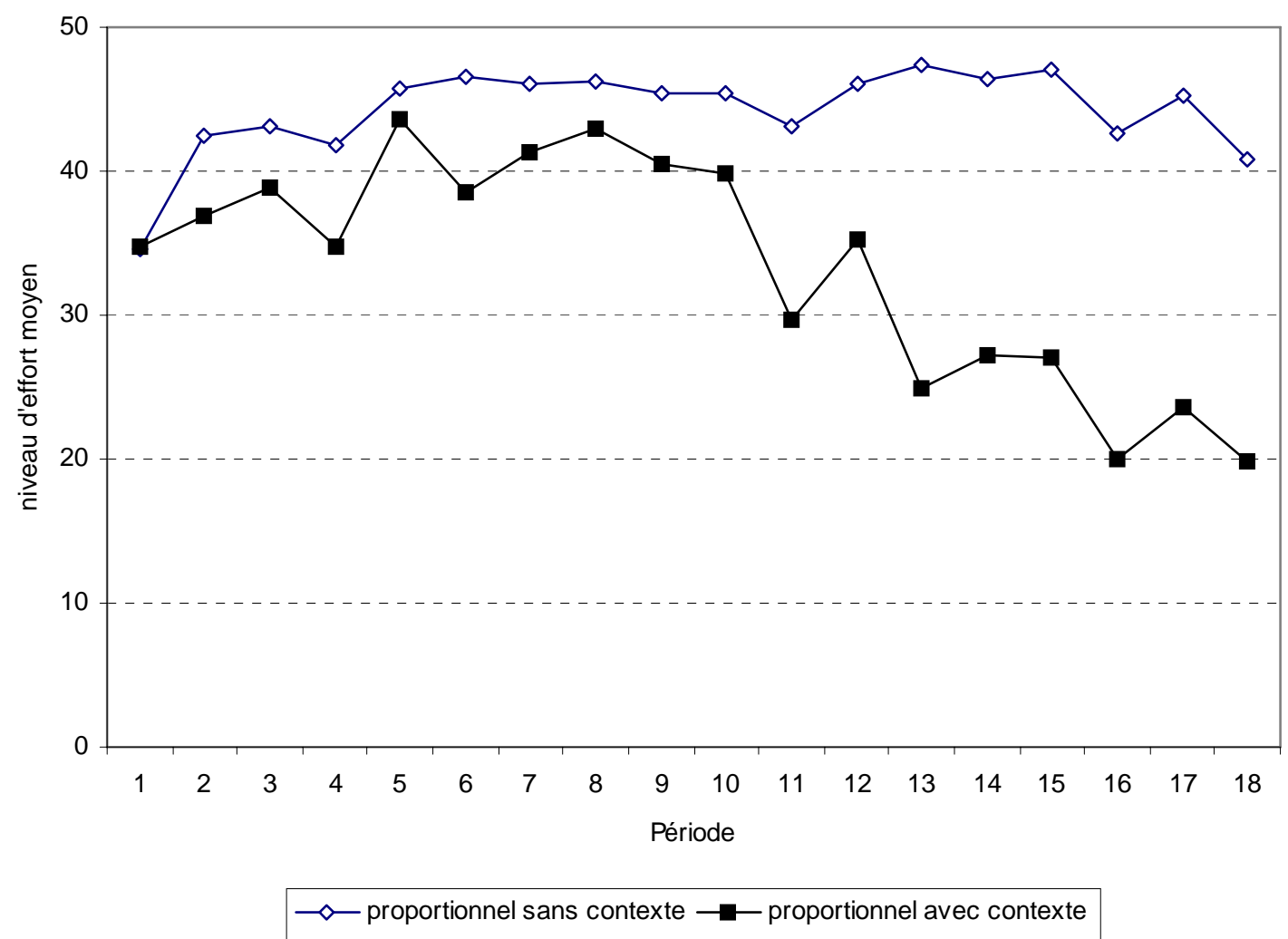

Le graphique 3 montre que le niveau d'effort en première période est identique dans les deux traitements. Ainsi le nombre de tâches réalisées sans erreur est de 34,65 tâches dans le système proportionnel sans contexte et de 34,83 tâches dans le système avec contexte. Un test non paramétrique de Mann-Whitney confirme qu'il n'existe aucune différence significative entre les deux traitements lors de la première période $(z=0,675)$. Si l'on considère maintenant 
l'ensemble des périodes, il apparaît que le niveau d'effort se maintient au-dessus de 40 unités dans le système proportionnel sans contexte tandis qu'il diminue fortement dans le système proportionnel avec contexte pour atteindre un niveau inférieur à 20 unités en dernière période. Ainsi le nombre moyen de tâches réalisées sans erreur sur l'ensemble des périodes est de 30,90 tâches dans le traitement avec système proportionnel avec contexte contre 44,21 dans le traitement avec système proportionnel sans contexte. Un test de Mann-Whitney réalisé sur moyennes individuelles indique que le niveau d'effort moyen individuel est significativement plus élevé dans le système proportionnel sans contexte que dans le système proportionnel avec contexte au niveau de confiance de $5 \%(z=1,989)$.

Le graphique 3 montre que le contexte fiscal influence négativement l'offre de travail des individus. Comment alors expliquer un tel effet? Une raison possible est que les individus qui ont été confrontés dans le passé à des taux d'imposition plus faible que 50\% et donc à une plus forte rémunération nette d'impôts seraient incités à réduire leur offre de travail dès lors qu'ils sont confrontés à un taux de 50\%. Cette explication est d'autant plus plausible que la probabilité d'avoir été confronté dans le passé à un taux plus avantageux que 50\% est plus élevée que l'inverse, compte tenu de la distribution de taux envisagée et que cette probabilité s'accroît au cours du temps ${ }^{22}$. Une autre explication possible réside dans le fait que l'expérience de taux plus forts dans le passé conduirait à une déshabitude à l'effort. Ainsi un individu qui aurait subi un taux à $79 \%$ et qui réduirait son offre de travail en conséquent, ne serait pas incité à la modifier par la suite ${ }^{23}$. Rappelons que l'enjeu principal de cette étude n'est pas tant d'étudier comment le contexte affect l'offre de travail mais davantage démontrer que le contexte fiscal n'est pas neutre et qu'il peut influencer dans un sens comme dans l'autre l'offre de travail.

En résumé, nos résultats mettent en exergue l'importance du contexte et soulignent que celui-ci peut avoir des effets désincitatifs sur l'offre de travail dès lors que les agents ont expérimenté dans le passé des taux plus attractifs.

\subsection{Une analyse paramétrique du rôle du contexte et du choix du système d'imposition}

\subsubsection{Les déterminants de l'offre de travail}

Etudions tout d'abord les déterminants de la probabilité que les participants ne fournissent aucun effort. La proportion de décisions sans effort est-elle plus élevée dans un 
système proportionnel avec contexte? Pour répondre à cette question précise, nous présentons dans le tableau 2 les résultats d'un moindre carré linéaire en probabilité où la valeur observée prend la valeur 1 lorsque la décision d'un participant B est d'aucune tâche réalisée correctement et 0 sinon. Le modèle linéaire en probabilité a été préféré au Probit vu le nombre peu élevé d'observations sans effort. ${ }^{24}$

Tableau 2

Déterminants des décisions sans effort

\begin{tabular}{l|cc}
\hline \hline Variable & $(1)$ & $(2)$ \\
\hline \hline Système & 0,0588 & 0,05601 \\
proportionnel avec & $(3,86)$ & $(3,69)$ \\
contexte & & \\
Système progressif & 0,0288 & 0,02327 \\
& $(3,08)$ & $(2,44)$ \\
& & $-0,008707$ \\
Productivité & & $(-2,93)$ \\
& & \\
Constante & $-6,59 \mathrm{e}-17$ & 0,07589 \\
& $(-0,00)$ & $(2,84)$ \\
\hline \hline $\bar{R}^{2}$ & 0,0190 & 0,0247 \\
\hline Nombre & 966 & 966 \\
d'observations & & \\
\hline \hline
\end{tabular}

Le système proportionnel sans contexte est la référence. Les valeurs du t-statistique sont entre parenthèses.

La première spécification du modèle considère l'impact de la progressivité et du contexte sur le niveau d'effort réalisé. Le système de référence est le système proportionnel sans contexte. La variable "système progressif" est une variable dichotomique qui prend la valeur 1 si le système est progressif et 0 autrement. La variable "contexte" est construite de façon similaire. La deuxième spécification intègre une variable supplémentaire dans l'analyse : la productivité des individus. Cette dernière variable caractérise l'habileté du participant à effectuer les tâches. La productivité du participant est mesurée par le nombre moyen de tâches réalisées au cours des périodes de la session expérimentale divisé par le temps moyen pris par le participant pour réaliser les tâches. Cette mesure idiosyncratique correspond à un effet individuel dans les régressions. Les résultats économétriques confirment largement les résultats précédents. Ils 
indiquent que relativement au système proportionnel sans contexte, le système proportionnel avec contexte et le système progressif incitent davantage les participants à ne fournir aucun effort. Les résultats de la deuxième spécification montrent que l'on obtient des résultats identiques en contrôlant la productivité des individus.

Le tableau 3 présente les déterminants de l'offre de travail des participants sous différents contextes institutionnels et leurs réactions aux différents systèmes de taxation. Comme nous avons observé des situations où le participant $\mathrm{B}$ refuse, sous certaines conditions, de faire des tâches alors que sous d'autres conditions il réalise le nombre maximal de tâches possibles, nous avons opté pour un modèle économétrique du type Tobit pour tenir compte des éléments de censure vers le haut et le bas. Quatre spécifications ont été retenues. Les deux premières spécifications concernent toutes les observations et les troisième et quatrième spécifications isolent les résultats du premier jeu. Seules les variables relatives à la fiscalité sont retenues dans les spécifications (1) et (3). La variable productivité est intégrée dans les spécifications (2) et (4) afin d'expliquer certaines différences dans les comportements des travailleurs lors de nos sessions expérimentales. 
Tableau 3. Offre de travail (Tâches réalisées correctement)

\begin{tabular}{|c|c|c|c|c|}
\hline Variable & $(1)$ & $(2)$ & (3) Jeu 1 & (4) Jeu 1 \\
\hline $\begin{array}{l}\text { Proportionnel } \\
\text { avec contexte }\end{array}$ & $\begin{array}{l}-2,395 \\
(-0,45)\end{array}$ & $\begin{array}{l}-2,914 \\
(-0,54)\end{array}$ & $\begin{array}{l}-6.925 \\
(-1.17)\end{array}$ & $\begin{array}{l}-5,887 \\
(-1,01)\end{array}$ \\
\hline Progressif & $\begin{array}{l}-12,11 \\
(-7,01)\end{array}$ & $\begin{array}{l}-11,27 \\
(-6,46)\end{array}$ & $\begin{array}{l}-3.943 \\
(-1.03)\end{array}$ & $\begin{array}{l}-1,905 \\
(-0,50)\end{array}$ \\
\hline $\begin{array}{c}\text { Interaction : } \\
\text { période*contexte }\end{array}$ & $\begin{array}{l}-1,569 \\
(-3,26)\end{array}$ & $\begin{array}{l}-1,470 \\
(-3,05)\end{array}$ & & \\
\hline Période & $\begin{array}{c}-0,08659 \\
(-0,53)\end{array}$ & $\begin{array}{c}0,08692 \\
(0,53)\end{array}$ & & \\
\hline Productivité & & $\begin{array}{l}1,560 \\
(2,92)\end{array}$ & & $\begin{array}{l}3,188 \\
(2,89)\end{array}$ \\
\hline Constante & $\begin{array}{c}53,73 \\
(26,45)\end{array}$ & $\begin{array}{l}40,25 \\
(4,99)\end{array}$ & $\begin{array}{l}46,281 \\
(16.30)\end{array}$ & $\begin{array}{r}18,48 \\
(1,87)\end{array}$ \\
\hline $\begin{array}{c}\text { Log de la } \\
\text { vraisemblance }\end{array}$ & $-2821,68$ & $-2817,38$ & -526.59 & $-522,43$ \\
\hline $\begin{array}{c}\text { Nombre } \\
\text { d'observations }\end{array}$ & 966 & 966 & 162 & 162 \\
\hline $\begin{array}{l}\text { Censures à } \\
\text { gauche }\end{array}$ & 19 & 19 & - & - \\
\hline Censures à droite & 399 & 399 & 55 & 55 \\
\hline
\end{tabular}

Le système proportionnel sans contexte est la référence.

Les valeurs du t-statistique sont entre parenthèses.

Les résultats pour l'ensemble des observations montrent que le système progressif conduit systématiquement à une réduction statistiquement significative du nombre de tâches effectuées correctement, relativement au système proportionnel sans contexte. La variable d'interaction 'période*contexte' montre également qu'un joueur qui a expérimenté au cours de l'expérience des taux de taxation plus avantageux que le taux de 50\%, tend à réduire son niveau d'effort, relativement à une situation où il a été confronté au taux de 50\% durant toute l'expérience. On constate finalement que les participants montrant une forte productivité réalisent davantage de tâches. En résumé, les résultats montrent bien que la dimension institutionnelle de la fiscalité importe sur l'offre de travail. 
Les résultats des spécifications concernant le jeu 1 confirment les observations des graphiques 1 et 3. Ces résultats montrent que le niveau d'effort lors du premier jeu est sensiblement le même quel que soit le traitement joué. Ainsi c'est bien le fait d'avoir expérimenté par le passé d'autres taux qui influence les décisions individuelles et non pas le seul fait de connaître l'existence d'autre taux de taxation comme c'est le cas dans le premier jeu.

Le tableau 4 présente les résultats des régressions sur les rentrées fiscales à l'aide d'un Tobit avec un seul point de censure.

\section{Tableau 4. Déterminants des rentrées fiscales}

\begin{tabular}{l|c}
\hline \hline Système & $\begin{array}{c}\text { Toutes les } \\
\text { observations }\end{array}$ \\
\hline \hline progressif & $\begin{array}{c}-412,36 \\
(-6,98)\end{array}$ \\
& $-577,28$ \\
Proportionnel & $(-6,01)$ \\
avec contexte & 2210,99 \\
Constante & $(51,90)$ \\
\hline \hline Log de la & $-7776,85$ \\
vraisemblance & 966 \\
Nombre & 19 \\
d’observations & \\
Censurée à 0 & \\
\hline \hline
\end{tabular}

Le système proportionnel sans contexte est la référence. Les valeurs du t-statistique sont entre parenthèses.

Le tableau 4 confirme les résultats de la section précédente. En effet, il indique que les rentrées fiscales diminuent avec la progressivité du système ainsi qu'en présence d'un contexte fiscal défavorable, relativement au système proportionnel sans contexte. Un test du ratio du maximum de vraisemblance indique que la différence de rentrées fiscales obtenues entre le système progressif et le système proportionnel avec contexte n'est significative qu'au seuil de confiance de $10 \%$. 


\section{Conclusion}

Plusieurs enseignements ressortent de notre étude. Tout d'abord, nos résultats expérimentaux montrent que le choix d'un système fiscal n'est pas neutre sur l'offre de travail. Ainsi nous observons, dans un cadre décontextualisé qu'offre l'expérimentation, que les effets désincitatifs sur l'offre de travail sont plus importants sous un système progressif que sous un système proportionnel. Par ailleurs, nos résultats indiquent que les rentrées fiscales sont également significativement plus importantes dans le traitement avec système proportionnel sans contexte que dans le traitement avec système progressif. Un autre résultat intéressant de notre étude est que le contexte fiscal affecte également les comportements individuels. En effet, pour un taux donné, les participants choisissent leur offre de travail en tenant compte de leurs expériences fiscales précédentes. En d’autres termes, si la concurrence fiscale n’est jamais mise en doute lorsqu'il s'agit de taxes touchant le capital, le choix du système de taxation touchant le travail est également sous contraintes de ce qui a été expérimenté par les contribuables.

A ce stade, il convient toutefois de nuancer nos propos. D'une part, nos résultats peuvent être conditionnés par le choix du design expérimental utilisé dans cette étude. En effet, comme tout protocole expérimental, notre design repose sur une réalité très simplifiée. Ainsi, à l'instar des études précédentes desquelles nous nous inspirions étroitement (Weck-Hannemann, 2003 LévyGarboua, Masclet et Montmarquette, 2007a, 2007b), il existe une asymétrie dans les rôles des individus puisqu'un seul un des deux participants travaille. De même, notre design expérimental ne considère que la redistribution du joueur $B$ vers le joueur $A$, ce qui supprime une partie de complexité du système de redistribution.

Il convient également d'être prudent quant à l'interprétation de nos résultats. En effet, si l'incitation des systèmes fiscaux est devenu à partir des années 90 une préoccupation essentielle des pouvoir publics, l'arbitrage incitation-protection n'ai pas forcément aisé. En effet, s'il s'avère que le système proportionnel génère davantage de rentrées fiscales et semble donc plus efficace fiscalement, un tel système peut à certain égard être perçu comme relativement injuste, en particulier pour les travailleurs les moins qualifiés, s'il ne réduit pas les écarts de revenus en prélevant des taxes sur les revenus les plus élevés pour verser des allocations aux revenus les plus bas. Par ailleurs, ce système fiscal doit garantir que le "travail paie", en particulier pour les moins qualifiés susceptibles de tomber dans les "trappes à pauvreté" que la mise en place d'un système proportionnel risquerait potentiellement d'accentuer.Dès lors, comment concilier 
efficacité fiscale et justice sociale? Autrement dit, efficacité fiscale est-elle forcément incompatible avec plus de justice sociale? Il semble qu'au contraire, ces deux critères soient loin d'être antagonistes. En effet, un rôle majeur dévolu aux gouvernements est de redistribuer les revenus dans la population. Or cette capacité de redistribution dépend aussi des rentrées fiscales et de la croissance économique. L'efficacité fiscale est donc nécessaire aux gouvernements qui recherchent la croissance économique via le travail et un maximum de rentrées fiscales. Des rentrées fiscales importantes permettent aux gouvernements d'offrir à leur population respective une gamme de services et de biens publics, alors que la croissance économique augmente la richesse collective. Le choix du système fiscal à retenir est donc une question majeure de finances publiques. Dès lors, le choix d'un système proportionnel avec taux unique, s'il offre davantage de recettes fiscales, peut être acceptable dès lors qu'il permet une plus grande redistribution des ressources afin d'atténuer les inégalités, ce taux unique pouvant d'ailleurs être relativement élevé pour permettre aux gouvernements d'atteindre un objectif important de redistribution.

Plusieurs extensions possibles de cette étude seraient intéressantes et permettraient d'apporter plus de réalisme à l'étude actuelle. Ainsi nous pourrions introduire la possibilité d'une redistribution bilatérale entre deux ou plusieurs travailleurs. De façon plus générale, lever les hypothèses d'asymétrie entre les joueurs permettrait de gagner en réalisme et permettrait d'introduire d'autres éléments qui ne sont actuellement pas pris en compte dans l'étude actuelle. 


\section{Notes}

${ }^{1}$ Cette moyenne recouvre de fortes disparités : les Etats-Unis et le Japon ne recouvrent qu'environ 30\% de leur PIB tandis que le ratio atteint $50 \%$ voir plus dans certains pays (en Suède notamment).

2 (1985, pp 19-34) a montré qu'une augmentation des taxes conduit à une substitution du travail taxé vers le travail non taxé (activités illégales, travail au noir). Henry (1983) estime que 90 milliards de dollars américains de revenus échappent annuellement à l'imposition aux États-Unis. Fortin, et al (1996) ont évalué pour leur parte, qu'en 1993, l'économie souterraine au Québec (excluant les activités criminelles) représente un minimum de 0,93\% du P.I.B., soit 1,49 milliards de \$. Les revenus déclarés sont également sensibles à une variation des taxes. Saez (1999), Feldstein (1995) et Gruber et Saez (2000) ont observé une augmentation significative du revenu déclaré imposable lorsque les taux de taxation diminuent.

${ }^{3}$ Les individus n'ont pas toujours la possibilité de choisir leur quantité de travail. Toutefois, les diverses forces économiques (conjoncture, évolution des techniques, réglementation) amènent souvent les employeurs à signer des contrats de travail pouvant refléter les préférences des salariés. Par ailleurs, un certain nombre d'emplois, comme par exemple le travail indépendant, offre aux individus une plus grande flexibilité concernant leur offre de travail.

${ }^{4}$ En effet, une population plus âgée aurait davantage de possibilité de réduire le nombre d’heures de travail effectuées, voir même de quitter son emploi pour une retraite anticipée. En ce qui concerne les travailleurs indépendants, ces derniers, n'étant pas soumis à un contrat qui réglemente précisément le nombre d'heures à effectuer, peuvent davantage faire varier leur offre de travail en fonction de la fiscalité en vigueur. Par ailleurs, les travailleurs indépendants auraient davantage d'occasions de pratiquer l'évasion fiscale ou de masquer une partie des revenus perçus, compte tenu de la nature même de leur activité. Enfin, la sensibilité au changement de taxation des individus percevant de hauts revenus s'explique en partie par leur accès privilégié à différents mécanismes permettant de changer la forme sous laquelle est perçu l'impôt (avantages en nature, "stock plans").

${ }^{5}$ S'il existe un certain nombre d'études économétriques qui ont étudié l'incidence de la taxation sur l'offre de travail, les revenus mais également sur la fraude fiscale, l'évasion fiscale et le travail illégal, Swenson (1988) souligne toutefois que ces études font généralement ressortir un certain nombre de problèmes majeurs : i) des erreurs de mesures ; ii) des biais de sélection ; iii) une mauvaise spécification de la fonction d'utilité ; iv) le non contrôle des variables.

${ }^{6}$ En effet, contrairement aux études précédentes, dans l'expérience de Sutter et Weck-Hannemann (2003), le taux de taxation n'est pas choisi par l'ordinateur mais au contraire par un participant à l'expérience. L'expérience de Sutter et Weck-Hannemann se déroule de la façon suivante : tout d'abord selon le traitement, les sujets sont informés ou pas de leur rôle (sujet A ou B). Ensuite, le sujet A choisit quel effort (sur un intervalle entre 0 et 40 unités), il souhaite réaliser pour chaque taux de taxation possible sur un intervalle continu de 0 à $100 \%$. Dans un deuxième temps, le sujet $\mathrm{B}$ choisit le taux de taxe parmi les taux proposés. Son choix détermine l'effort que le sujet A devra réaliser à la fin de l'expérience.

${ }^{7}$ Swenson (1988) observe quant à lui que les rentrées fiscales sont maximisées au taux de 73\%. Au-delà de ce taux, les rentrées fiscales diminuent car les sujets réduisent fortement leur effort de production.

${ }^{8}$ Aux États-Unis, la question a souvent été abordée ces dernières années ; c'était par exemple le cheval de bataille de Jerry Brown, candidat démocrate malheureux à la présidence en 1992.

${ }^{9}$ Ainsi, sous l'ancien Régime au XVIIIe siècle, en France, seul le tiers état payait le Vingtième puis le Dixième tandis que le clergé et l'aristocratie en étaient exemptés. Cette différence d'imposition fut d'ailleurs l'une des causes de la révolution française.

${ }^{10}$ L'impôt est proportionnel au revenu lorsque chaque citoyen verse le même pourcentage de son revenu; l'impôt progressif est un impôt non proportionnel pour lequel la part de prélèvement augmente avec le revenu de chaque contribuable.

${ }^{11}$ Comme le soulignent Kahneman, Knetsch et Thaler, 1986, les individus sont souvent amenés à comparer leur situation actuelle avec celles qu'ils ont expérimentées par le passé en se prenant eux même pour référence dans le temps (Kahneman, Knetsch et Thaler 1986).

${ }^{12}$ Ces résultats confirment les travaux antérieurs de Phelps (1973), Sadka (1976) et Seade (1977).

${ }^{13}$ Le sujet A est nécessaire pour que le sujet B soit conscient que l'argent prélevé sur le revenu de son travail n'est pas "brûlé" mais effectivement bien redistribué. 
${ }^{14}$ Les joueurs A et B disposent de revues et de jeux afin d'éviter une possible identification des rôles joués par chacun des participants. Toutefois, ces alternatives en loisir ne peuvent pas influencer les décisions du participant $\mathrm{B}$ dans la mesure où la durée de l'expérience n'est pas fixée au préalable mais est, au contraire, conditionnée par les décisions des participants $B$.

${ }^{15}$ Les instructions du jeu sont lues à haute voix aux participants de chaque session expérimentale. Les instructions sont disponibles sur demande.

${ }^{16}$ Comme dans les expériences précédentes ( Swenson, 1988; Sillamaa, M.A, 1999a, b et c et Sutter et WeckHannemann, 2003), la répartition des taches à effectuer se confond avec la répartition des revenus puisque chaque tâche réalisée sans erreur est rémunérée à l'identique (soit 100 unités expérimentale par tâche). Ainsi l'intervalle correspondant aux joueurs réalisant entre 31 et 52 tâches correspond à la tranche de revenu pour laquelle le taux d'imposition est de 79\% (tranche de revenu entre 3100 et 52000 unités).

${ }^{17}$ Ces taux renvoient à cinq situations différentes. $12 \%$ reflète un taux de prélèvement minimum en dessous duquel il est impensable d'avoir une politique de redistribution. $28 \%$ correspond à un taux moyen de taxation qui touche un grand nombre de travailleurs. Le taux à $50 \%$ touche une part de la population moins grande mais qui semble davantage flexible en terme de variation de son offre de travail. Enfin le taux à $79 \%$ est un taux élevé mais qui représente en réalité un certain nombre de nouveaux travailleurs renonçant à un soutien social pour accepter du travail, voyant de ce fait leur taux marginal implicite de taxation augmenter de façon considérable. Ces taux sont ceux qui sont généralement retenus dans la littérature expérimentale (Swenson, 1988, Sillamaa 1999a, b ; LévyGarboua, Masclet et Montmarquette, 2005a, 2005b).

${ }^{18}$ L'ordre d'appariation des systèmes a été programmé afin de nous assurer d'un nombre suffisant d'observations du taux à $50 \%$ pour chacune des périodes.

${ }^{19}$ Si un effet "contexte" existe, alors nous pouvons présager que ce dernier devrait vraisemblablement affecter négativement les décisions des individus pour un même taux à 50\% compte tenu de l'existence de deux taux en dessous de $50 \%$ et d'un seul taux au dessus.

${ }^{20}$ Au delà de l'effet des expériences précédentes, susceptible d'influencer les décisions des participants, nous pouvions également présager que la seule information donnée en début d'expérience sur l'existence de systèmes fiscaux alternatifs puisse également affecter les choix des individus. La comparaison du premier jeu avec les jeux précédents permet d'isoler ces deux effets.

${ }^{21}$ La diminution du niveau d'effort en dernière période ne peut pas être imputée à un "effet de fin de jeu" dans la mesure où les participants ne sont pas informés de la durée de l'expérience.

${ }^{22}$ En effet, les conséquences du contexte sur l'offre de travail dépendent évidemment de la distribution des taux qui peut être plutôt favorable (défavorable) au taux de 50\%. Ainsi la distribution de taux suivante : 28, 50, 79 et $85 \%$ aurait sans aucun doute eu un effet "contexte" positif sur l'offre de travail pour le taux de 50\%.

${ }^{23}$ Notre design expérimental ne permet pas d'isoler clairement cet effet de l'effet en terme d'habitude à une rémunération nette d'impôt plus forte. Toutefois un premier élément de réponse consiste à comparer le nombre de tâches réalisées sans erreur dans le traitement avec contexte selon que le taux précédent était plus élevé ou plus faible que 50\%. Si l'explication en terme de déshabitude à l'effort est juste, nous devrions observer que l'offre de travail dans le traitement sans contexte soit plus élevé que l'offre de travail dans le traitement avec contexte pour un taux à $50 \%$ après avoir expérimenté un taux plus fort dans le passé. Le nombre de tâches réalisées sans erreur pour $50 \%$ dans le traitement avec contexte et après avoir été confronté à un taux de $79 \%$ à la période précédente ( 41,12 tâches) n'est pas significativement différent de celui obtenu sans contexte (p>0,10). Au contraire, le nombre de tâches réalisées lorsque le participant a été confronté à la période précédente à un taux de $12 \%$ ou $28 \%$ est significativement plus faible, soit 29,96 tâches. Ces résultats ne nous permettent donc pas de valider l'hypothèse relative à l'existence d'un effet lié à la déshabitude à l'effort à cause de l'expérience de taux plus forts dans le passé.

${ }^{24}$ Le nombre d'observations sans effort étant relativement faible, nous avons opté pour un modèle linéaire en probabilité plutôt qu'un modèle de type probit. Par ailleurs, la variable "productivité" qui est introduite dans la deuxième spécification du modèle est mesure idiosyncratique qui permet par ailleurs de prendre en compte l'effet individuel. Nous avons également vérifié que le nombre d'observation avec aucune tache correspond bien, sauf rares exceptions à des individus différents à chaque fois. Il s'agit au total de 19 observations nulles sur 966 dont 13 observations dans le traitement progressif et 6 observations dans le traitement proportionnel. 


\section{Bibliographie}

Ashworth, J. and Ulph, D. (1981), "Estimating Labour Supply with Piecewise Linear Budget Constraints", Edition Brown, London.

Cowel, F.(1985), “Tax Evasion with Labor Income” Journal of Public Economics,26 (1): 19-34

Feldstein, M. (1995), "The Effect of Marginal Tax Rates on Taxable Income: A Panel Study of the 1986 Tax Reform Act”, Journal of Political Economy 103, 551-572.

Fortin, B., Garneau, G., Lacroix, G. Lemieux,T. et Montmarquette, C. (1996), "Léconomie souterraine au Québec: Mythes et réalités", Québec : Les Presses de l’Université Laval, Québec.

Fortin, B. Joubert N. et G. Lacroix ' Offre de travail au noir en présence de la fiscalité et des contrôles fiscaux', Économie et prévision, à paraître.

Gruber, J. and Saez, E. (2000), "The Elasticity of Taxable Income: Evidence and Implications", NBER, working paper.

Hausman J. (1981), "Labor Supply", in H Aaron and J Pechman eds, in How Taxes Affect Economic Activity (Washington DC).

Henry J. (1983), "Non Compliance with US Tax Law Evidence of Size, Grow and Composition", working paper.

Kahneman, D., Knetsch J.L. and Thaler R.H. (1986), "Fairness as a Constraint on Profit Seeking : Entitlement in the Market", American Economic Review, 76, pp728-41.

Lévy-Garboua L. Masclet D. et Montmarquette C. (2007a), " Fiscalité et offre de travail : évidence expérimentale ", Economie et Prévision, à paraître

Lévy-Garboua L. Masclet D. et Montmarquette C. (2007b), "A Micro-foundation for the Laffer Curve In a Real Effort Experiment", manuscript non publié.

Phelps E. S. (1973), "Taxation of wage income for economic justice", Quarterly Journal of Economics, 87: 331-354.

Sadka E. (1976), "On income distribution, incentive effects and optimal income taxation, Review of Economic Studies, 9, 216-267.

Seade JK. (1977), "On the Shape of Optimal Tax Schedules, Journal of Public Economics, 23, 351-366.

Saez E. (1999), "The Effect of Marginal Tax Rates in Income: A Panel Study of Bracket Creep”, NBER WP 7367.

Schelling T. (2007), 'The strategy of conflict', Cambridge : Harvard University Press. 
Sillamaa, M.A (1999a), “Taxpayer Behavior in Response to Taxation: Comment and New Experimental Evidence”, Journal of Accounting and Public Policy 18, 165-177.

Sillamaa, M.A (1999b), "How Work Effort Responds to Wage Taxation: A non Linear versus a Linear Tax Experiment”, Journal of Economic Behavior and Organization, 39, 219-2333.

Sillamaa, M.A (1999c), "How Work Effort Responds to Wage Taxation : an Experimental Test of a Zero Top Marginal Tax Rate”, Journal of Public Economics, 73, 125-134.

Sillamaa, M.A. and M. Veal (2000), “The Effects of Marginal Tax Rates on Taxable Income: A Panel Study of the 1988 Tax Flattening in Canada”, Research Report no 354.

Slemrod, J. (1996), "What is the Simplest Tax System of them All? Economic Effects of Fundametal Tax Reform", Edition Brookling Institution Press, Washington DC.

Slemrod, J. (1998),"A General Model of the Behavioural Response to Taxation", NBER working paper.

Sutter, M. and H. Weck-Hannemann, (2003), “Taxation and the Veil of Ignorance: A Real Effort Experiment on the Laffer Curve”, Public Choice 115, 217-240.

Swenson, C. (1988), “Taxpayer Behavior in Response to Taxation: an Experimental Analysis”, Journal of Accounting and Public Policy 7, 1-28. 